\title{
Cerebral Tuberculosis in an Active-duty Military Officer That Led to Tinnitus From a TB Brain Abscess; A Case Report; Case Report.
}

smart asare ( $\sim$ sasare@email.davenport.edu )

Detroit Medical Center https://orcid.org/0000-0002-2620-1308

Bello Figuim

Neurosurgery HCY

Aubin Sandio

Wayne State University School of Medicine

Ngouatna Serge

Cameroon Baptist Convention Health Board: Cameroon Baptist Convention Health Service

\section{Tamkam Cecillia}

Cameroon Hospital

Ignatius Esene

Department of Neurosurgery

Bitang L.J.

Neurosurgery department CURY

Mark Moisi

Detroit Medical Center Neurosurgery department

Fankam Casimir

Neurosurgery Department CURY

\section{Research}

Keywords: Tuberculosis, Tinnitus, Abscess, Military Officer, Convulsive crises

Posted Date: November 10th, 2021

DOl: https://doi.org/10.21203/rs.3.rs-1010546/v1

License: (c) (1) This work is licensed under a Creative Commons Attribution 4.0 International License.

Read Full License 


\section{Abstract}

Tuberculosis has been a pertinent public health problem for both developing and developed nations. For developed nations, military personal has the higher risk since they do travel to developing nations to embark on their duties. Cerebral tuberculosis is rare and if they occur, they tend to manifest as either meningitis or tuberculoma. Tinnitus is more likely in men particularly persons exposed to loud noises along with other causes. We present a case of cerebral tuberculosis induced tinnitus in a 26-year-old male army officer who presented with a one-week episode of convulsive crises and loss of consciousness after being exposed to a loud noise while on duty. Cranial MRI showed right temporo-parietal and left parietal finger-like hyper-signals with edema on Flair and T2. However, brain CT-scan showed right parietotemporal and left parietal sub cortical hypodensities and finger-like borders without contrast re-uptake. There was strong suspicion for TB brain abscess leading to a possible manifestation of tinnitus in this patient.

\section{Case presentation}

A 26-year-old male army officer presented with chief complaints of convulsive crisis and loss of consciousness when he heard a loud noise while on duty. He fell to the ground and was found unconscious and drooling by a colleague. He was immediately transferred by non-medical means to our hospital for management. The patient experienced rigidity as well as uncontrolled muscle spasms leading to jerky motions which lasted for about one to two minutes and occurred two hours before admission in a non-febrile context. The convulsive crises occurred two hours prior to admission in a nonfebrile state. The patient was then worked up for review of systems (ROS)-SpO2 was 98\%; RR was 24 $\mathrm{cpm}, \mathrm{BP}=125 / 91 \mathrm{mmHg}$, Pulse $=103 \mathrm{bpm}$, Glasgow coma scale $=15 / 15$, isochoric iso-reactive pupils, blood sugar $=1,11 \mathrm{~g} / \mathrm{l}$; Temperature $=37^{\circ} \mathrm{C}$. No motor or sensitive deficits, no meningeal signs, no former convulsive crisis, there was symmetry for chest movements, no signs of respiratory distress, resonant percussion sounds. Also, there was no urine incontinence, dysuria, scrotal swelling and external genitalia deformations. No peripheral lymph nodes (cervical, axillary, inguinal) were palpable.

Upon checking the labs, WBC: 5.05, HGB: $12.4 \mathrm{~g} / \mathrm{l}, \mathrm{PLT}: 313,000$ electrolyte panel reveals all normal except moderate hypomagnesemia. HIV 1 and 2 serology was negative, Cardiovascular examination shows PPP, audible heart sounds at all four auscultation points, no MGR and RRR. On respiratory exam; there were no signs of distress, no tracheal deviation, resonant to percussion, CTAB and no CVAT. On abdominal exam; no HSM and normal bowel movement and sounds. Finally, Neurological; no acute distress (NAD), AAOx3, CN 2-12 intact, MME is normal, recall is $3 / 3$, coordination and concentration intact, follows command and no motor or sensory deficits. Did bronchi fibroscopy along with bronchoalveolar lavage, in search of TB by PCR.

\section{Conclusion}

Tinnitus remains the second most prevalent service-connected disability. Patients with cerebral TB abscess are at increased risk for this condition. 


\section{Introduction}

Tuberculosis is one of the most well researched infectious diseases because of its' mortality and morbidity burden that it has posed on human lives since the days of stone age [1]. Since 1932, the respiratory system is the most common organ system initially and latterly involved [2]. Pulmonary Tuberculosis (TB) that leads to brain abscess can greatly mimic central nervous system (CNS) tumors, posing as a challenge in terms of accurate diagnosis and management hence affecting prognosis. $\mathrm{M}$. tuberculosis causes rare forms of brain abscesses with most occurring from disseminated tuberculosis or individuals from areas where tuberculosis is endemic [3]. TB affects the CNS in three common patterns; meningitis, tuberculomas, and finally as spinal arachnoiditis [4]. Headache and fever seem to be the most common symptoms for individuals with CNS TB which can progress to coma if diagnostic delay creeps in or is left untreated [5-7]. CNS TB can look like or mimic a malignant CNS tumor clinically and hence posing as a diagnostic conundrum $[8,9]$. Pulmonary TB has to be considered first and almost every organ can suffer the burden of TB [10].

As of 2014 , there have been approximately 9.6 million tuberculosis cases worldwide with reports estimating 1.5 million deaths as a result of TB infection globally [10]. CNS-TB makes up about $1 \%$ of all tuberculosis cases worldwide and has the highest mortality than any other forms of the disease manifestation as far as organ systems are concerned [11]. This pathogen; mycobacterium tuberculosis, invades the central nervous system following primary infection through inhalation of droplets. This gets filtered by regional lymph nodes. Secondary insult or non-proper management can lead to seeding into blood stream. As a result of reactivation, the organism travels to CNS via the blood stream with inflammation of the meninges being the most often manifestation [12]. CNS-TB can also affect nerves leading to cranial nerve paresis as well as hydrocephalus [13]. There are a handful of complications that can occur with TB affected the lungs and then later spreading to other organs. Though rare, CNS-TB usually occurs in immunocompetent unvaccinated patients, or patients who are non-compliant with the medications but travel and work in endemic developing countries [14] particularly manifesting as CNS-TB abscess induced tinnitus as in our patient.

\section{Discussion}

Air-borne and close contacts are the main routes of tuberculoses transmission. Transmission typically tend to occur when a person or persons inhale(s) droplet nuclei containing M. tuberculosis. They could be rare modes of transmission as well. Droplet nuclei usually traverse the nasal passages or mouth then into the upper respiratory tract, and the bronchi to finally get to the alveoli of the lungs. Tinnitus affects people differently. It is more prevalent in males. For some people, this condition can significantly affect quality of life with most patients having symptoms like fatigue, stress, trouble concentrating, headaches and problems with work and family life- all of which our patient reported. Our patient had no known chronic medical pathologies, was never hospitalized but had a year history of recurrent mild parieto-occipital headaches relieved by over-the-counter analgesics. He has never been operated upon, does not smoke and does not take alcohol, has no known food or drug allergies, up to date on vaccinations since 
childhood, and no cough. He has history of several travels to developing countries as well as a one-year community life in a formation camp.

Our patient possibly had an infection with Mycobacterium tuberculosis that was not properly managed, allowing the bacteria to travel through the bloodstream to infect other organs and tissues. Our patient did not have miliary TB as only his brain suffered this disease burden. Though the bacteria usually travel to the meninges of the brain and or spinal cord and affects them leading to meningeal tuberculosis, our patient was worked up and showed no signs and symptoms or imaging to suspect meningitis. High blood pressure and malformed blood vessels was likely the reason for the spread of the bacteria to the brain. These blood flow changes make tinnitus more noticeable. We also considered tuberculoma which has some connecting symptoms like fever, decreased consciousness, headaches and nausea and vomiting as well as motor deficits, seizures and neck stiffness plus even signs of infarction. Intracranial tuberculomas may be solitary or multiple. Solitary tuberculomas may be indistinguishable from cranial abscess or primary brain tumor hence it is necessary to rule out tuberculoma in patients with intracranial pressure and mass lesions. However, imaging studies proved otherwise, ruling out either of these conditions.

Work-up for toxoplasmosis, primary CNS lymphoma, meningitis, tuberculoma, and encephalitis were all negative. TAP CT-Scan showed bilateral interstitial pneumopathy with left apical cavern, associated with pulmonary nodules and coelio-mesenteric plus para-aortic adenopathies. On direct smear, there was absence of alcohol-acidio-resistant bacteria. Bronchi expectorations using PCR GeneXpert detected mycobacteria tuberculosis. Results of bronchi fibroscopy showed moderate diffuse inflammation of left and right bronchi and widening of the sub-segmental eperon from dorsal to culminal regions of the left principal bronchi. Treatment for TB was based on multidisciplinary approach where we Started treatment of multi-focal tuberculosis for 2 months and continued with Rifampicin: $15 \mathrm{mg} / \mathrm{kg} / \mathrm{day}$, Isoniazide: $10 \mathrm{mg} / \mathrm{kg} /$ day, Pyrazinamide: $35 \mathrm{mg} / \mathrm{kg} /$ day, Ethambutol: $20 \mathrm{mg} / \mathrm{kg} /$ day for a duration of 12 months.

Finally, the images from the patient that helped us arrive at our definite diagnosis.

\section{Conclusion}

Tinnitus remains the second most prevalent service-connected disability. Patients with cerebral TB abscess are at increased risk for this condition. Positive bacillary Pulmonary tuberculosis in an immune competent patient is fairly common among campers or military officers who travel to developing countries without proper vaccinations. Those vaccinated but constantly exposed like military officers on duty are also at risk. Proper management with quadruple therapy with anti-tuberculosis medications for about six to twelve months is the standard treatment.

\section{Abbreviations}

WBC; White Blood Cell 
PPP; Peripheral Pulses Present,

TB; Tuberculosis

MGR; Murmurs, Gallops, \& Rales

HGB; Hemoglobin

RRR; Regular Rate Rhythm

PLT; Platelet

CVAT; Costovertebral Angle Tenderness

CTAB; lungs are Clear To Auscultation Bilaterally

MME; Mini Mental Exam

CN 2-12; cranial nerves 2-12

AAOx3; Alert, Awake, Oriented.

\section{Declarations}

Funding; this study is and has not been funded by anyone school, organization, or any parties. (nonapplicable).

Conflict of Interest: The authors declare that they have No Conflict of Interest. (non-applicable)

Complies with ethical standards.

Consent to participate (applicable)

Consent for publication (applicable)

Availability of data and material (applicable).

Code availability (non-applicable).

Author's contributions;

Data collection process;

1. Reference's list and proof reading; Dr Fankem Casimir

2. Patient seen as inpatient/outpatient at Detroit Medical Center, Detroit, Michigan; (Dr. Mark Moisi, Dr. Aubin Sandio, Dr. Smart Asare) 
3. Abstract, discussion, conclusion; (Dr. Smart Asare, Dr TAMKAM CECILLIA, Dr. Bello Figuim, Dr Ngouatna Serge, and Dr. Bitang L.J).

4. Introduction, Case Presentation; (Dr. Ignatius Esene, Dr. Smart Asare, Dr. Aubin Sandio).

\section{References}

1. Pezzella, A. T. (2019). History of pulmonary tuberculosis. Thorac Surg Clin.;29(1):117. [PubMed] [Google Scholar]

2. Poulsen, A. (1957). Some clinical features of tuberculosis. Acta Tuberc Scand. 33(1-2):37-92.; concl. [PubMed] [Google Scholar]

3. Mathiesen, G.E., \& Johnson, J.P. (1997). Brain abscess. Clin Infect Dis. 25: 763-79. [Google Scholar] [PubMed]

4. Al-Deeb, S. M., Yaqub, B. A., Motarey, K. R. (1992). Neurotuberculosis: A review. Clin Neurol Neurosurg. 94(Suppl.): S30-33. [PubMed] [Google Scholar]

5. Farinha, N. J., Razali, K. A., Holzel, H., et al. (2000). Tuberculosis of the central nervous system in children: A 20-year survey. J Infect.;41(1):61-68. [PubMed] [Google Scholar]

6. Hinman, A. R. (1967). Tuberculous meningitis at Cleveland Metropolitan General Hospital 1959 to 1963. Am Rev Respir Dis.;95(4):670-73. [PubMed] [Google Scholar]

7. Kennedy, D. H., Fallon, R. J. (1979). Tuberculous meningitis. JAMA. 1979;241(3):264-68. [PubMed] [Google Scholar]

8. Chaudhry, U. R., Farooq, Rauf. F., Bhatti, S. K. (2011). Tuberculosis simulating brain tumour. Neuroradiol J.;24(3):350-56. [PubMed] [Google Scholar]

9. Suslu, H. T., Bozbuga, M., \& Bayindir, C. (2011). Cerebral tuberculoma mimicking high grade glial tumor. Turk Neurosurg. 2011;21(3):427-29. [PubMed] [Google Scholar]

10. Pai, M., Nicol, M. P., \& Boehme, C. C. (2016). Tuberculosis diagnostics: State of the art and future directions. Microbiol Spectr:;4(5) [PubMed] [Google Scholar]

6. Marxreiter, F., Winkler, J., Uhl, M., \& Madžar, D. (2017). A Case Report of Severe Delirium after Amantadine Withdrawal. Case reports in neurology, 9(1), 44-48. Retrieved from: https://doi.org/10.1159/000460814

7. Branas P, et al. (2000). Treatments for fatigue in multiple sclerosis: a rapid and systematic review. Health Technol Assess. 4(27):1-61. [PubMed] [Google Scholar] 
8. Hadjimichael O, et al. Fatigue characteristics in multiple sclerosis: The North American Research Committee on Multiple Sclerosis (NARCOMS) survey. Health Qual Life Outcomes. 2008; 6:100. doi: 10.1186/1477-7525-6-100. [PMC free article] [PubMed] [CrossRef] [Google Scholar]

9. Tellez $\mathrm{N}$, et al. Fatigue in multiple sclerosis persists over time: a longitudinal study. $J$ Neurol. 2006;253(11):1466-70. doi: 10.1007/s00415-006-0247-3. [PubMed] [CrossRef] [Google Scholar]

10. Finlayson $M$, Preissner $K$, Cho $C$. Outcome moderators of a fatigue management program for people with multiple sclerosis. Am J Occup Ther. 2012;66(2):187-97. doi: 10.5014/ajot.2012.003160. [PubMed] [CrossRef] [Google Scholar]

11. World Health Organization: Global Tuberculosis Report (2016). World Health Organization, Geneva, Switzerland. Available from:

http://www.who.int/tb/publications/global_report/en/9789241565059_eng.pdf

12. H.M. Peto., R.H. Pratt., T.A. Harrington., et al, (2009). Epidemiology of extrapulmonary tuberculosis in the United States, 1993-2006, Clin. Infect. Dis. 49 1350-1357.

13. R.B. Rock., M. Olin., C.A. Baker., et al, (2008). Central nervous system tuberculosis: pathogenesis and clinical aspects, Clin. Microbiol. Rev. 21 243-261.

14. Y. Amin., A. Shaukat., B.A. Mian., (2004). Intracranial manifestations of tuberculosis: an imaging study, Biomedica $201-4$.

\section{Supplementary Files}

This is a list of supplementary files associated with this preprint. Click to download.

- floatimage5.jpeg

- 1.jpg 